\title{
Chemical Analysis of the Glomeruli from the Kidney of Diabetics and Subjects with Choronic Glomerulonephritis
}

\author{
Tokutaro Sato, Shiro Oguma, * Takao Saito and \\ KaORU Yoshinaga \\ The Second Department of Internal Medicine and *the First \\ Department of Pathology, Tohoku University School of \\ Medicine, Sendai 980
}

\begin{abstract}
Sato, T., Oguma, S., Satto, T. and Yoshinaga, K. Chemical Analysis of the Glomeruli from the Kidney of Diabetics and Subjects with Chronic Glomerulonephritis. Tohoku J. exp. Med., 1980, 132 (2), 207-212 — A method was established to isolate the glomeruli from the kidney fixed in formaldehyde. O-Hydroxylysylglycosides and hydroxyproline in the isolated glomeruli from the kidney of normal subjects, diabeties and subjects with chronic glomerulonephritis were measured on the alkaline hydrolyzate of the glomeruli. O-Hydroxylysylglycosides in 1000 glomeruli were $9.42 \pm 2.68$ nmoles (mean \pm s.D.) for controls, $12.92 \pm 7.36$ nmoles for diabetics and 12.54 \pm 4.62 nmoles for subjects with chronic glomerulonephritis. Hydroxyproline in 1000 glomeruli from control, diabetics and subjects with chronic glomerulonephritis was $43.0 \pm 10.7,82.0 \pm 39.3$ and $52.1 \pm 21.3$ nmoles, respectively. Hydroxyproline in the hyalinized glomeruli from diabetics was 123.3 \pm 22.4 nmoles/1000 glomeruli, and significantly increased in comparison with that in the glomeruli from chronic glomerulonephritis or control subjects. glomeruli; O-hydroxylysylglycosides; hydroxyproline; diabetes; chronic glomerulonephritis
\end{abstract}

The glomerular basement membrane appears to play a vital physiological role as a selective filter. Chemical analysis of the glomerular basement membrane has been carried out to obtain information in regard to its structural alteration in pathological conditions such as diabetes mellitus and glomerulonephritis (Beisswenger and Spiro 1973; Westberg and Michael 1973a, b; Kefalides 1974; Sato et al. 1975; Galle and Machieu 1975). There is, however, no general agreement concerning the changes in the chemical compositions of the glomerular basement membrane in these diseases. The reason for this may be the differences of cases studied, and there is also a possibility that the fraction of the glomerular basement membrane obtained from the diseased glomeruli are not identical from study to study. It is necessary to analyze the chemical compositions of the whole glomeruli as well as the glomerular basement membrane.

Krakowa and Greenspon (1951) introduced a method to isolate the glomerular basement membrane from fresh or frozen kidneys. In this study, we established a method to isolate glomeruli from kidneys of diabetics and subjects with chronic

Received for publication, August 18, 1979. 
glomerulonephritis fixed in formaldehyde, and analyzed O-hydroxylysylglycosides and hydroxyproline in the glomeruli.

\section{Materials and Methods}

Materials. The formaldehyde-fixed kidneys from autopsy materials or removed for renal transplantation were used. An average age of the control group was 55.3 years, and the kidneys showed no pathological abnormality. Mean age of 11 diabetics was 67.6 years, of which 4 cases showed hyalinization in 52 to $87 \%$ of the glomeruli, and 7 cases showed mild changes of diffuse type. Mean age of 8 cases of chronic glomerulonephritis was 22.4 years, of which 4 cases histologically showed marked hyalinization of the glomeruli.

Isolation of the glomeruli. The procedure to isolate the glomeruli from the formaldehyde-fixed kidneys is shown in Fig. 1. The formaldehyde-fixed renal cortex, about $2 \mathrm{~g}$, was cut into small pieces, and then forced to pass through a $115 \mathrm{mesh}$ sieve $(7.5 \mathrm{~cm}$ in diameter) with the bottom surface of a beaker. The material which emerged through the sieve was poured on the sieves of 80 and 200 mesh in succession. The glomeruli fraction was obtained on the 200 mesh sieve, from which the glomeruli were picked up and collected under a stereomicroscope.

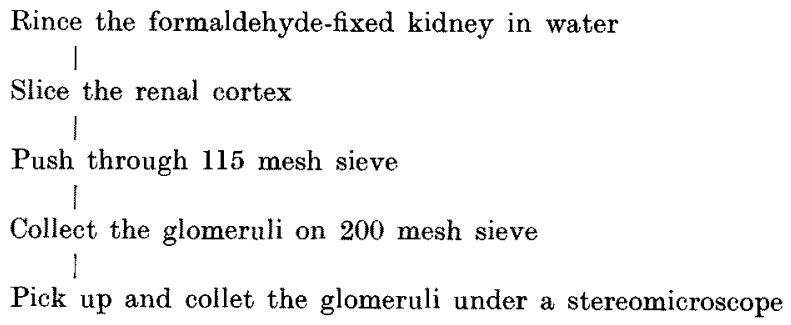

Fig. 1. Procedure to isolate the glomeruli from the formaldehyde-fixed kidney.

Analysis of the compositions of the glomeruli. To one thousand glomeruli were added $0.5 \mathrm{ml}$ of $2 \mathrm{~N} \mathrm{NaOH}$, and the mixture was heated at $105^{\circ} \mathrm{C}$ for $20 \mathrm{hr}$. The hydrolyzate was titrated with $2 \mathrm{~N} \mathrm{HCl}$ to bring the $\mathrm{pH}$ to $2.5-3.0$ and then applied to a column $(1.4 \times 4$ $\mathrm{cm})$ of Dowex $50 \times 4\left(\mathrm{H}^{+}\right.$form $)$. The column was washed exhaustively with water. The remaining substances adsorbed on the column were eluted with $1.5 \mathrm{~N} \mathrm{NH} \mathrm{NH}_{4} \mathrm{OH}$. The eluate was evaporated to dryness in vacuo, and the residue was dissolved in $2.0 \mathrm{ml}$ of water. The content of hydroxyproline in the eluate was determined by the method of Kivirikko et al. (1967). Aliquots of the samples were hydrolyzed in $2 \mathrm{~N} \mathrm{HCl}$ at $100^{\circ} \mathrm{C}$ for $4 \mathrm{hr}$. In order to determine the content of $\mathrm{O}$-hydroxylysylglycosides, the hydrolyzate was analyzed for glucose by the use of glucose oxidase (Bergmeyer and Bernt 1970)

The content of protein in the glomeruli was determined by the method of Lowry et al. (1951) on the unhydrolyzed materials using bovine serum albumin solution as a standard.

\section{Results}

The glomeruli could not be purified by sieving technic alone, so we picked up the glomeruli under a stereomicroscope to achieve better isolation (Fig. 2).

The amounts of protein, hydroxyproline and O-hydroxylysylglycosides of the glomeruli are shown in Table 1. Mean of the protein content of 1000 glomeruli was $52.5 \mu \mathrm{g}$ for controls, $62.0 \mu \mathrm{g}$ for diabetics and $69.5 \mu \mathrm{g}$ for subjects with glomerulonephritis. Significant differences $(p<0.01)$ in the protein content was observed 


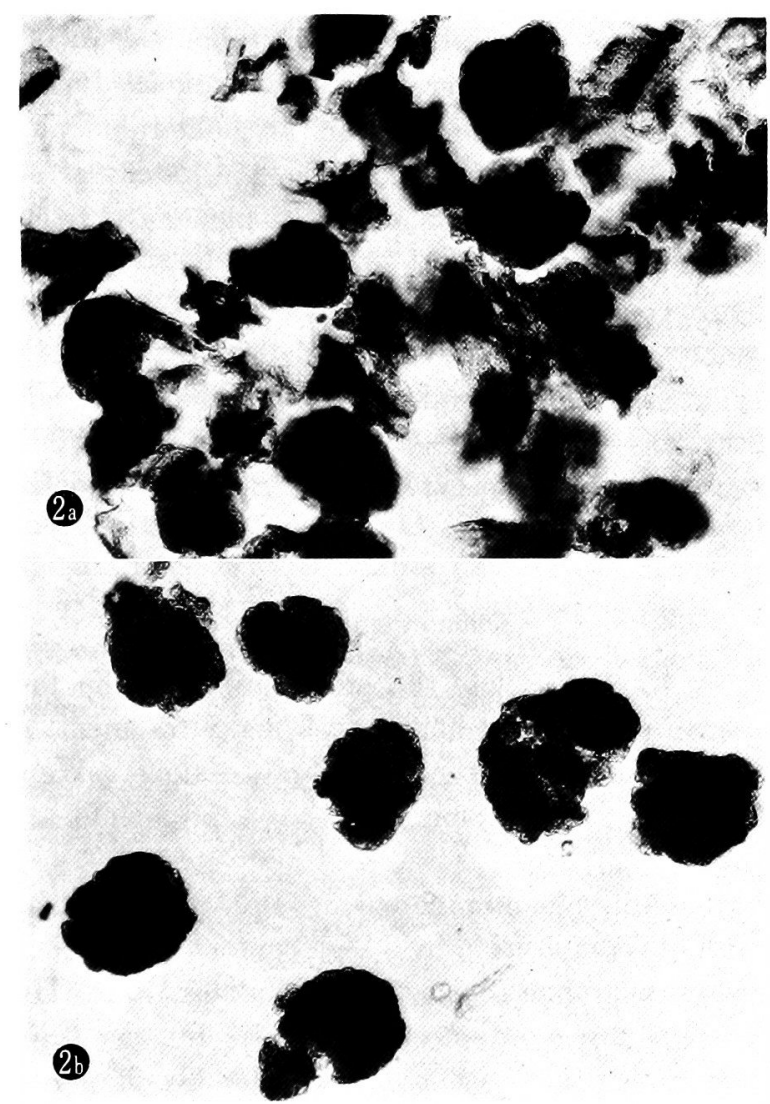

Fig. 2. Photomicrographs of the isolated glomeruli. a: Glomeruli fraction on 200 mesh sieve. b: Purified glomeruli.

TABLE 1. The content of O-hydroxylysylglycosides and hydroxyproline in the glomeruli

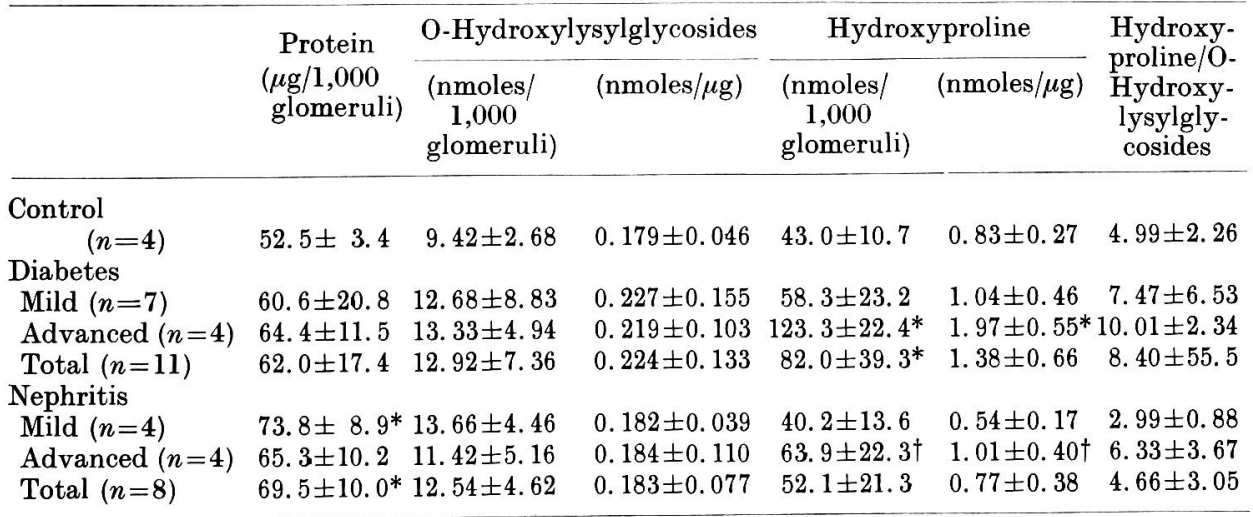

Mean \pm S.D.

* Significantly different from the control value $(p<0.01)$.

$\dagger$ Significantly different from the value for advanced diabetes $(p<0.05)$. 
between the control and the glomerulonephritis group. Mean of the O-hydroxylysylglycosides content in 1000 glomeruli was 9.42 nmoles for the control, 12.93 nmoles for the diabetics and 12.54 nmoles for the glomerulonephritis group. The content of hydroxyproline in the glomeruli from the kidney of advanced diabetic nephropathy was 123.3 nmoles, and significantly higher than that of control and advanced glomerulonephritis.

The ratio of hydroxyproline to O-hydroxylysylglycosides was $4.99 \pm 2.26$ (mean \pm S.D.) for the control, $8.40 \pm 5.55$ for the diabetics and $4.66 \pm 3.05$ for the glomerulonephritis group. The number of the glomeruli showing advanced diabetic nephropathy was especially high in the diabetic group, but no significant difference was detected in the ratio between the diabetics and the control or the glomerulonephritis group $(p>0.05)$.

\section{Discussion}

In this study, we tried to isolate the glomeruli from the formaldehyde-fixed kidney. The glomeruli were contaminated with many fragments of the connective tissue and could not be purified by sieving technique alone. We could picked up, under a stereomicroscope, 1000 glomeruli free of fragments of the connective tissue in about $3 \mathrm{hr}$.

The glomerular basement membrane belongs to the collagen family of proteins, and is characterized in its composition by a high content of glycine, the occurence of hydroxylysine and hyroxyproline (Spiro 1967; Kefalides 1971). Hydroxylysine and O-hydroxylysylglycosides are more effective markers for the basement membrane than hydroxyproline, since they occur in considerably larger amounts in the membrane than in fibrillar collagens (Spiro 1969). In contrast, hydroxyproline is found in almost equal amount in the basement membranes when compared with collagens (Spiro 1967).

Analysis for the chemical compositions for the glomerular basement membrane revealed that in diabetics the hydroxyproline content increases by $6.5-10.95 \%$, and the hydroxylysine content increases by 3.6-19.4\% over the control (Beisswenger and Sprio 1973; Westberg and Michael 1973a; Kefalides 1974; Sato et al. 1975). Analysis of the whole glomeruli by Klein et al. (1975) and Beisswenger and Spiro (1973), suggests that, in the glomeruli from diabetics, the increment of hydroxyproline is larger than that of hydroxylysine; Klein et al (1975) reported that the hydroxyproline content of the glomeruli from diabetics increased by $36 \%$ over the control, while Beisswenger and Spiro (1973) reported that the hydroxylysine content of the glomeruli from diabetics increased by $14 \%$ over the control. These results suggest that analysis of the chemical compositions of the isolated glomerular basement membrane may only partially reflect the changes of the glomeruli in diabetes mellitus.

In this study, hydroxyproline in the glomeruli from diabetics increased significantly $(p<0.01)$ as compared with that from control subjects. The change was especially remarkable in the hyalinized glomeruli, and the ratio of hydroxyproline to 
0-hydroxylysylglycosides was much larger in the glomeruli from the diabetics than those from the control, which is different from the changes observed in the basement membrane (Beisswenger and Spiro 1973; Westberg and Michael 1973a; Kefalides 1974; Sato et al. 1975) and suggests that not only basement membrane but collagenous fiber increases in the diabetic glomeruli.

The chemical composition of the basement membrane isolated from the human kidney of glomerulonephritis cases was studied by Westberg and Michael (1973b), and Galle and Machieu (1975). Westberg and Michael (1973b) reported that the content of hydroxyproline and hydroxylysine appeared to be reduced in chronic glomerulonephritis and idiopathic membranoproliferative glomerulonephritis, and the ratio of hydroxyproline to hydroxylysine in these groups, 3.3, was slightly lower than that in the control group (4.04). On the other hand, Galle and Machieu (1975) reported that the content of hydroxyproline and hydroxylysine was almost same in both of the control and glomerulonephritis groups. In our study, the content of hydroxyproline and $\mathrm{O}$-hydroxylysylglycosides in the glomeruli of the glomerulonephritis groups did not differ significantly from the counterpart values in the control.

The hyalinized glomeruli from the diabetics contain much larger amount of hydroxyproline than those from the glomerulonephritis group (Table 1), suggesting that deposits in the diabetic glomeruli have more collagenous materials, and the process of hyalinization is different in the two diseases.

\section{References}

1) Beisswenger, P.J. \& Spiro, R.G. (1973) Studies on the human glomerular basement membrane. Diabetes, 22, 180-193

2) Bergmeyer H.U. \& Bernt, E. (1970) In Methoden der enzymatischen Analyse. Weinheim/Bergstrasse, Verlag Chemie, p. 1172.

3) Galle, P.\& Machieu, P. (1975) Electrodense alteration of kidney basement membranes. Amer. J. Med., 58, 749-764

4) Kefalides, N.A. (1971) Isolation of a collagen from basement membranes containing three identical a-chain. Biochem. biophys. Res. Commun., 45, 226-234

5) Kefalides, N.A. (1974) Biochemical properties of human glomerular basement membrane in normal and diabetic kidneys. J. clin. Invest., 53, 403-407

6) Kivirikko, K.I., Latien, O. \& Prockop, D.J. (1967) Modification of a specific assay for hydroxyproline in urine. Anal. Biochem., 19, 249-255

7) Klein, L., Butche, D.L., Sudilovsky, O., Kikkawa, R. \& Max Miller (1975) Quantification of collagen in renal glomeruli isolated from human nondiabetic and diabetic kidneys. Diabetes, 24, 1057-1065

8) Krakowa, C.A. \& Greenspon, S.A. (1951) Localization of the nephrotoxic antigenic components of kidney tissue. Brit. J. exp. Path., 34, 27-34

9) Lowry, O.H., Rosebrough, N.J. \& Parr, A.L. (1951) Protein measurement with the Folin phenol reagent. J. biol. Chem., 193, 265-275

10) Sato, T., Munakata, H., Yoshinaga, K. \& Yosizawa, Z. (1975) Comparison of the chemical composition of glomerular and tubular basement membranes obtained from kidneys of diabetics and nondiabetics. Clin. chim. Acta, 61, 145-150

11) Spiro, R.G. (1967) Studies on the renal glomerular basement membrane: Preparation and chemical composition. J. biol. Chem., 242, 1915-1922

12) Sprio, R.G. (1969) Characterization and quantitative determination of the hydroxylysine-linked carbohydrate units of several collagens. J. biol. Chem., 244, 602-612 
13) Westberg, N.G. \& Michael, A.F. (1973a) Human glomerular basement membrane: Chemical composition in diabetes mellitus. Acta med. scand., 194, 39-47

14) Westberg, N.G. \& Michael, A.F. (1973b) Human glomerular basement membrane. Acta med. scand., 194, 49-57 